\title{
Associations confessionnelles et dynamique de développement. Analyse des pratiques marchandes et financières des associations confessionnelles à l'Est de la République Démocratique du Congo
}

Thèse de Doctorat (PhD), Louvain-la-Neuve (Belgique), Université Catholique de Louvain (UCL), 2014

\section{Emmanuel Musongora Syasaka}

\section{OpenEdition}

Journals

Édition électronique

URL : http://journals.openedition.org/anthropodev/331

DOI : 10.4000/anthropodev.331

ISSN : 2553-1719

Éditeur

APAD - Association pour l'anthropologie du changement social et du développement

Édition imprimée

Date de publication : 1 septembre 2014

Pagination : 201-203

ISBN : 9791093476018

ISSN : 2276-2019

Référence électronique

Emmanuel Musongora Syasaka, « Associations confessionnelles et dynamique de développement Analyse des pratiques marchandes et financières des associations confessionnelles à l'Est de la République Démocratique du Congo », Anthropologie \& développement [En ligne], 40-41 | 2014, mis en ligne le 01 septembre 2016, consulté le 24 septembre 2020. URL : http://journals.openedition.org/ anthropodev/331; DOI : https://doi.org/10.4000/anthropodev.331

La revue Anthropologie \& développement est mise à disposition selon les termes de la Licence Creative Commons Attribution 4.0 International. 


\title{
Associations confessionnelles et dynamique de développement \\ Analyse des pratiques marchandes et financières des associations confessionnelles à l'Est de la République Démocratique du Congo
}

\author{
Emmanuel Musongora Syasaka ${ }^{1}$ \\ Thèse de Doctorat (PhD), Louvain-la-Neuve (Belgique), \\ Université Catholique de Louvain (UCL), 2014
}

\section{Résumé}

Depuis quelques décennies, la République Démocratique du Congo (RDC) fait face à un foisonnement d'associations devenues omniprésentes dans le champ socioéconomique. Cette dynamique est à inscrire dans l'émergence des agents économiques qui surgissent de la base pour trouver des réponses aux différents problèmes auxquels font face les populations tant en milieu rural qu'urbain. Sous l'impulsion de certains leaders, les populations inventent diverses pratiques formelles et informelles pour assurer leur survie. Ces populations créent à leur manière des structures économiques remettant ainsi en cause le modèle de développement qui a prévalu dès la Seconde Guerre Mondiale. Les activités initiées peuvent être marchandes (production, distribution, petit commerce, microcrédit, coopératives) ou non marchandes (tontines, travaux communautaires, mise en place des infrastructures sociales). L'émergence de ces nouveaux acteurs dans l'espace privé et public est aussi une remise en question du modèle de gouvernance antérieure. Parmi ces acteurs figurent les associations confessionnelles qui connaissent une montée en puissance sur le plan socioéconomique.

En RDC, particulièrement dans la région de Butembo au Nord-Kivu, les églises font figure d'acteurs incontournables dans l'exercice des activités marchandes et non marchandes. Elles mettent en place une pluralité d'initiatives qui exigent de s'intéresser à la structuration des activités socioéconomiques qu'elles mènent et à leur mode de financement. Cette étude, à la suite de Karl Polanyi, s'inscrit dans la logique selon laquelle les activités socioéconomiques ne se réalisent pas seulement par le marché et l'Etat. Les associations dans leur diversité sont également actrices dans ce domaine. Elles présentent à ce propos une double nature. Alors qu'elles se définissent comme étant sans but lucratif, il se structure autour d'elles des activités

${ }^{1}$ E-mail : emmanuelmusongora@gmail.com 
marchandes et financières. Elles ont la qualité d'opérer en tant qu'acteurs hybrides dont la capacité d'innovation dépend en partie de leur capacité d'hybrider les types de ressources, en mobilisant d'un côté les ressources non monétaires et monétaires, et de l'autre les ressources marchandes et non marchandes en fonction des logiques de projet qu'elles entreprennent. Les associations confessionnelles organisent de manière permanente et professionnelle des activités marchandes et financières au point $d$ 'instituer une forme particulière de "business religieux ". Ces micro-initiatives sont réalisées dans des contextes complexes qui mettent en relation divers acteurs privés et publics, locaux/nationaux et internationaux, confessionnels et non confessionnels. La particularité des associations confessionnelles qui consiste à "s'autofinancer " en combinant diverses ressources favorise la création de divers liens sociaux et de réseaux au niveau local et transnational. Les interactions entre les acteurs entraînent des changements organisationnels voire institutionnels. La dynamique marchande et financière liée à ce " business religieux " local exige de se pencher sur une nouvelle analyse de la socio-économie des associations confessionnelles et leur contribution au développement local.

Dans cette étude, la question centrale est de savoir comment les initiatives marchandes et financières des associations confessionnelles produisent de l'impact sur le développement local ? Quatre questions spécifiques s'articulent autour de celle-ci :

- Quels sont les facteurs expliquant le foisonnement des associations confessionnelles et par quelles pratiques organisent-elles des activités marchandes et financières?

- Quels sont les acteurs impliqués dans ces pratiques, les interactions et les rapports entre eux?

- Comment ces initiatives marchandes et financières ont-elles un impact sur l'amélioration du cadre de vie au niveau local ?

- Quelle politique faut-il mettre en place pour rendre efficace les pratiques marchandes et financières exercées par les associations confessionnelles ?

La thèse que nous défendons dans cette analyse consiste à considérer que les associations confessionnelles ne peuvent être ignorées pour le rôle qu'elles jouent en tant qu'acteur socioéconomique. Pour mobiliser d'importantes sommes d'argent, elles intègrent dans leurs pratiques des logiques marchandes et financières réalisées aussi dans des sphères non confessionnelles. L'étude se veut une analyse de la socio-économie des associations confessionnelles afin de comprendre leur influence sur l'économie locale de la région de Butembo. D'une part, elle permet d'expliquer le mécanisme et le processus d'émergence d'une forme particulière du business religieux au sein des associations confessionnelles congolaises ainsi que la typologie des pratiques marchandes et financières qu'elles mettent en place. D'autre part, en partant d'une série d'activités socioéconomiques et 
d'études de cas réalisées sur cinq confessions religieuses, l'analyse se penche sur les interactions que ces pratiques marchandes et financières confessionnelles entraînent. Elle propose enfin une réflexion sur l'encadrement du secteur associatif confessionnel congolais afin de le rendre utile à l'économie nationale. 